\title{
Covid 19 patient hides his travel history; a case report in Pakistan
}

\begin{abstract}
Covid 19 is evolving as an epidemic. This is a case report of Covid 19 patient who had previous surgery for Carcinoma stomach. He was referred for follow-up CT chest and abdomen. The patient hides his recent travel history to Malaysia just to get his CT done. His HRCT findings were typical for CV-19 and later on his PCR confirmed CV-19 but he already came in contact with health care providers. Patients are concealing their travel histories which are also spreading infections.
\end{abstract}

Keywords: Covid 19, Radiologists, CT scan
Volume 7 Issue 3 - 2020

\author{
Shehzad Khan,' Muhammad Nadeem, ${ }^{2}$ Bakht \\ Rokhan, ${ }^{3}$ Shah Abbas, ${ }^{4}$ Sanyya $\mathrm{Hadi}^{5}$ \\ 'Consultant Radiologist, Saidu Teaching Hospital, Pakistan \\ ${ }^{2}$ Associate Professor of Surgery, Saidu Teaching Hospital, \\ Pakistan \\ ${ }^{3}$ Associate Professor of Radiology, Saidu Teaching Hospital, \\ Pakistan \\ ${ }^{4}$ Assisstant Professor of Surgery, Saidu Teaching Hospital, \\ Pakistan \\ ${ }^{5}$ Resident Radiology, AFIRI Rawalpindi, Pakistan
}

Correspondence: Shehzad Khan, Consultant Radiologist, Department of Radiology, Saidu Teaching Hospital, Pakisthan, Tel +923339474003, Email elegantradiologist@gmail.com

Received: April 2I, 2020 | Published: May 05, 2020

\section{Introduction}

The increasing number of CV-19 cases in Europe, USA and Asia has produced excessive burdens on hospitals, private clinics and healthcare providers. Drastic safety and precautionary measures have been adopted in the form of mass disinfectant sprays, use of gloves, masks and other personal protective gear. ${ }^{1}$ Government is mandating strict measures by imposing lockdowns in all provinces in Pakistan, ordering citizens to stay at their homes and allowing only one family member to go to groceries, pharmacies. The importance of hand washing, mask wearing and other precautions are being emphasized by community and religious leaders and educators. Hospitals in Pakistan are establishing their own protocols, i.e. closure of all elective procedures, surgeries and non-emergency outpatient visits. These precautions are put in place to insure there is no overcrowding or unnecessary spreading of the novel CV-19. As a result of the new, more restrictive precautions, patients with non-emergent medical problems have been untruthful or have exaggerated their symptoms in an effort to access treatment. These particular patients are not being forthright concerning their recent travel history and/or contacts. There were 4 recent cases from Srinagar, Kashmir ${ }^{2}$ in which patients concealed their travel histories and tested positive for CV19. Radiologists and technologists play a pivotal role in the Covid 19 pandemic, performing and reporting X-rays, CT scans and ultrasounds for infected patients. ${ }^{3}$ They face a high rate of routine exposure to the virus in the course of their work.

\section{Case}

A case reported in Swat, Pakistan, a patient was referred from the Emergency Surgical Unit to the Radiology Department for CT scans of the chest and abdomen. She had previous surgery for CA -Stomach. She was having dyspepsia, heartburn and epigastric discomfort. After full history was taken, the CT was performed and findings were that of pneumonia, possibly Covid-19. As shown in Figures 1-4. While the CT was being interpreted, the Radiologist contacted the patient's attendant to inquire about the patient's travel history. Relatives confirmed the patient had recently travelled to Malaysia. A nasopharyngeal swab was performed on the patient and sent for PCR which turned out to be COVID-19 positive. The two CT technicians were then quarantined for 14 days and other precautionary measures for infection control was done. ${ }^{4}$ The patient was referred to COVID -19 OPD in Saidu Teaching Hospital. But died a week later, his funeral was attended by approximately 200 people. Later that day, 16 of the attendees were determined to have Covid 19, and all were quarantined.

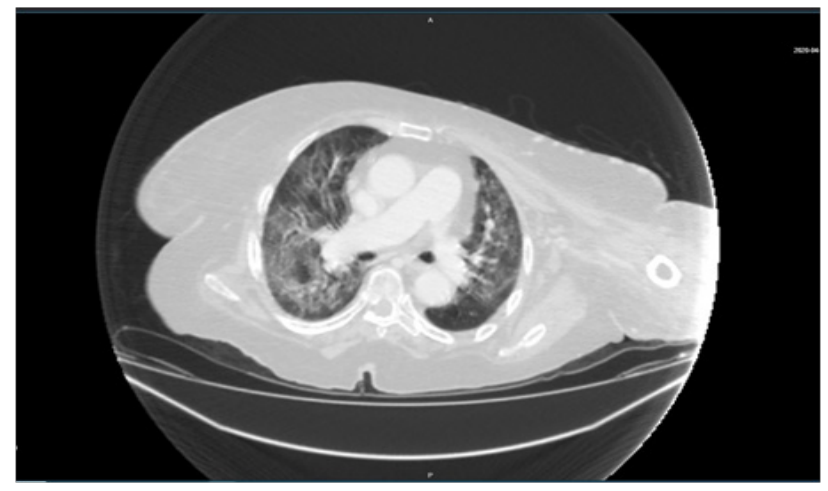

Figure I 55 year old female with no respiratory symptoms. Axial Section of HRCT shows bilateral involvement of lungs showing patchy and reticular opacities with Ground glass haze.

\section{Discussion}

The most important part of the case report is that in these intense conditions, patients wants to get there treatment done by hiding or giving incomplete history. Radiology staff should take comprehensive history before doing imaging or having some interventional procedures. Those coming in contact with the patients should use PPEs. 


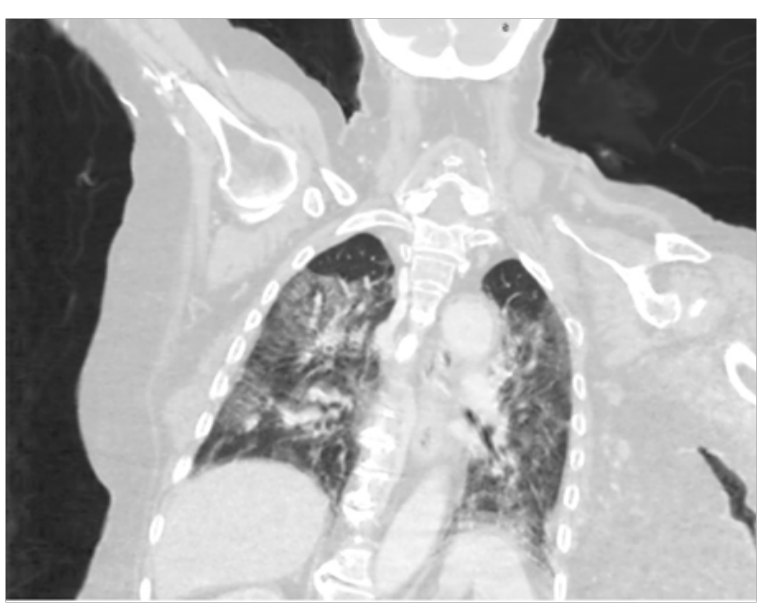

Figure 2 Coronal cut the same patient showing extensive involvement of all the lobes.

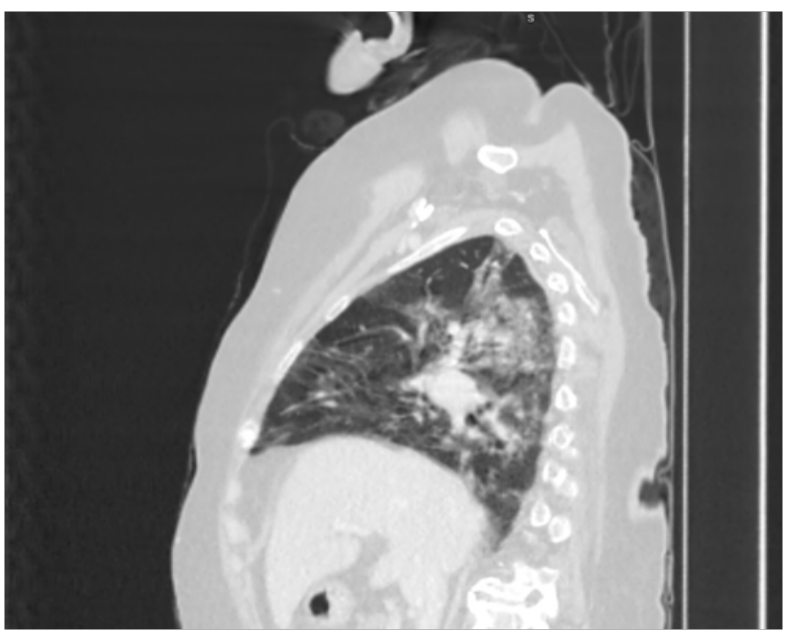

Figure 3 Sagittal slice if the right lung with patches.

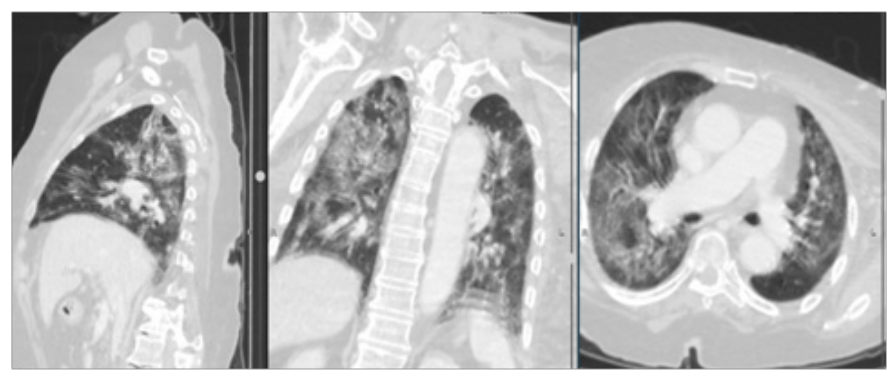

Figure 4 Sagittal, Coronal and Axial sections.

\section{Conclusion}

In conclusion, it is imperative that the relationships between health care professionals and patients have a high degree of truthfulness and trust in an environment that encourages such. It is important for the doctor to take a thorough history, obtain proper consent and counsel the patient in regard to their disease. Doctors must maintain patient confidentiality with the exception of issues that impact public health. On the other hand, the patient should not conceal any relevant information concerning their illness or history. This is particularly vital in the case of infectious diseases. The government should provide education to the public and create and enforce laws in an effort to prevent such deception.

\section{Acknowledgments}

None.

\section{Funding}

Self funding.

\section{Conflicts of interest}

Author declares that there is no conflict of interest.

\section{References}

1. Jinli Ding, Haihong Fu, Yaou Liu, et al. Prevention and control measures in radiology department for COVID-19. Eur Radiol. 2020;16:1-6.

2. COVID-19: 4 more test positive in Kashmir; Authorities fear more cases as people hide travel history. 2020

3. J C L Rodrigues, S S Hare, A Edey, et al. An Update on COVID-19 for the Radiologist - A British Society of Thoracic Imaging Statement. Clin Radiol. 2020;75(5):323-325.

4. Juan Yu, Ning Ding, Huan Chen, et al. Infection Control Against COVID-19 in Departments of Radiology. Acad Radiol. 2020;27(5):614-617. 